\title{
5 Das Wirken Gottes in De mortibus persecutorum
}

Nachdem sich die vorangehenden Kapitel vornehmlich mit der menschlichen Seite der Gewalt beschäftigt haben - mit dem Charakter, den Taten und den Strafen der menschlichen Gewalttäter -, soll nun die göttliche Seite der Gewalt in den Blick genommen werden. Es soll gezeigt werden, dass es sich bei der göttlichen Gewalt vornehmlich um reagierende Gewalt handelt. Es wurde bereits gezeigt, dass die Gewalt, die an den Tyrannen verübt wird, strafender Natur ist und in Übereinstimmung mit dem Charakter des jeweiligen Tyrannen beschrieben wird. In De mortibus persecutorum lassen sich grundsätzlich zwei Handlungsweisen Gottes ${ }^{1}$ unterscheiden: Entweder handelt Gott selbst, oder er lässt von ihm eingesetzte Principes handeln. Die Beispiele für beide Handlungsweisen sollen im Folgenden genauer analysiert werden. Wo es sinnvoll erscheint, sollen auch anthropologische Aussagen Laktanz' in die Betrachtung einbezogen werden, da es oft erst durch die Beziehungsbeschreibung zwischen Gott und Menschen möglich wird, Gottes Handeln zu beschreiben.

Zu Beginn des Werkes konstatiert Laktanz, dass das Leid der Christen unter den Verfolgungen nicht darin zu begründen sei, dass Gott nicht für seine Anhänger Sorge trage:

Die, die Gott beleidigt hatten, liegen darnieder, diejenigen, die den heiligen Tempel zerstört hatten, sind in größerem Sturz gefallen, diejenigen, die die Gerechten martervoll zerfleischten, haben unter himmlischen Schlägen und verdienten Qualen den schädlichen Lebensatem ausgehaucht. Spät geschah dies zwar, aber angemessen. Deren Strafen schob Gott nämlich auf, damit er an ihnen große und bewundernswerte Exempel statuiere, an denen die Nachwelt lernen könne, dass es nur einen Gott gebe und eben dieser auch als Richter den Übeltaten freilich entsprechende Strafen über die Gottlosen und Verfolger verhänge. ${ }^{2}$

Bereits im Proömium wird also deutlich, dass Gott strafend vorgeht. Um einen größtmöglichen Effekt zu erzielen, verschiebt er laut Laktanz die Strafen jedoch. Durch diese zeitverzögerte Reaktion ist es möglich, auch für die Zukunft solches

Anmerkung zu 5: In diesem Kapitel behandelte Textabschnitte: Lact. mort. pers. 1,5-6; 5,6-7; 31,1; $49,5-7 ; 8,4 ; 8,6 ; 14,4-5 ; 31,3-4 ; 36,4 ; 35,3 ; 2,5 ; 10,2 ; 3,2-3 ; 4,1-3 ; 5,1-6 ; 2,5 ; 10,2 ; 5,7$.

1 Es soll explizit nicht versucht werden, ein umfängliches Gottesbild aus De mortibus persecutorum zu konstruieren. Gott ist nahezu ausschließlich deus - nicht einmal deus christianorum. Seine Handlungen können greifbar gemacht werden. Siehe zur Problematik des ungreifbaren Gottesbildes beispielsweise auch Fitschen (2001), 64.

2 Qui insultaverant deo, iacent, qui templum sanctum verterant, ruina maiore ceciderunt, qui iustos excarnificaverant, caelestibus plagis et cruciatibus meritis nocentes animas profuderunt. sero id quidem, sed graviter ac digne. distulerat enim poenas eorum deus, ut ederet in eos magna et mirabilia exempla, quibus posteri disceret et deum esse unum et eundem iudicem digna videlicet supplicia impiis ac persecutoribus inrogare. Lact. mort. pers. 1,5-6 (Brandt/Laubmann 1897, 172,11-173,1; Creed 1897, 4,14-20). 
Fehlverhalten $\mathrm{zu}$ unterbinden. Das Bild eines strafenden Gottes ist dem Leser durchaus bekannt. In der paganen Literatur finden sich unzählige Beispiele von Gottheiten, die Menschen für ihre Verstöße gegen göttliches Recht bestrafen. ${ }^{3}$ Auch alttestamentarische Gottesvorstellungen umfassen den strafenden und richtenden Gott $^{4}$ und selbst das Neue Testament darf nicht vergessen werden. ${ }^{5}$ Die Frage, ob und wieso Gott strafe, behandelte Laktanz bereits in seinem Werk De ira Dei. Für ihn steht außer Frage, dass Gott, wenn er gütig sei, auch Zorn empfinden müsse:

Wenn aber Gott weder sich Mühe macht noch einem andern Mühe bereitet: Warum sollen wir dann nicht sündigen, so oft wir es der Kenntnis unserer Mitmenschen verheimlichen und die staatlichen Gesetze umgehen können? [...] vergreifen wir uns doch am fremden Gut, mit oder ohne Blutvergießen, wenn es außer den Gesetzen nichts weiter zu fürchten gibt! [...] - wie kann man die Religion selbst ohne Furcht erhalten und bewahren? Denn was man nicht fürchtet, das verachtet man, und was man verachtet, das verehrt man gewiß nicht. So kommt es, daß Religion, Majestät und Ehre auf Grund von Furcht besteht; Furcht gibt es aber nicht, wo keiner zürnt. Ob du also die Gnade oder den Zorn oder beides von Gott wegnimmst, du hebst damit notwendig die Religion auf, und ohne Religion ist das Menschenleben voll von Torheit, Verbrechen und Brutalität. Denn sehr zügeln kann das Gewissen den Menschen, wenn wir glauben wollten, unter den Augen Gottes zu leben, und meinten, daß nicht nur unser Tun von oben erblickt, sondern auch unser Reden und Denken von Gott gehört wird. ${ }^{6}$

Furcht vor der Strafe Gottes sei der Grund für Menschen, sich an Regeln und Gesetze zu halten. Gerade die Strafe tritt für Laktanz also in den Vordergrund und ist essentiell für sein Religionsverständnis und somit auch sein Menschenbild. Noch bevor die einzelnen Situationen beschrieben werden, in denen Gott Gewalt an den Tyrannen verübt, weiß der Rezipient, dass Folter und Schläge eingesetzt wurden. Es wird aber auch deutlich, dass diese nur gegen diejenigen gerichtet werden, die Gott, den Tempel oder die Gerechten angegriffen haben. Gott sieht sich also nicht gezwungen, gegen

3 In dieser Arbeit wurden bereits einige Beispiele genannt: Die mit Wahnsinn gestrafte Io (in dieser Arbeit Seite 50, besonders Anm. 36) oder Marsyas (in dieser Arbeit Seite 207). Diese lassen sich umfangreich ergänzen. Siehe auch Considine (1969); Irmscher (1950); Nebel (1951).

4 Man denke hier an Ps 45,7; Ps 72,2; Ps 76; Ps 96,13; Spr 28,5; Spr 29,7; Ex 22,23; Ex 32,10 -11; Dtn 11,16; Nah; Neh 13,18; Ez 7,14; Hos 5,10; so gibt es auch unzählige Literatur zu dem Thema. Eine kurze Auswahl: Janowski (2013); Berges (2004); Jeremias (2009).

5 Beispielsweise Apg 19,5; Joh 5,22; Joh 16,7-11; Lk 10,14; Mt 11,24; Mt 12,36; Kor 5,10; Joh 5,29; Apg 17,31; Röm 2,16; Röm 14,10; Epheser 6,8.

6 Quod si negotium deus nec habet nec exhibet, cur ergo non delinquamus, quotiens hominum conscientiam fallere licebit ac leges publicas circumscribere? ubicumque nobis latendi occasio adriserit, consulamus rei, auferamus aliena vel sine cruore vel etiam cum sanguine, si praeter leges nihil est amplius quod verendum sit. [...] quomodo religio ipsa sine metu teneri aut custodiri potest? quod enim non metuitur, contemnitur, quod contemnitur, utique non colitur. ita fit ut religio et maiestas et honor metu constet; metus autem non est ubi nullus irascitur. sive igitur gratiam deo sive iram sive utrumque detraxeris, religionem tolli necesse est, sine qua vita hominum stultitia scelere inmanitate conpletur. multum enim refrenat homines conscientia, si credamus nos in conspectus dei vivere, si non tantum quae gerimus videre desuper, sed etiam quae cogitamus aut loquimur audiri a deo putemus. Lact. ira. 8,5-8 (Kraft/ Wlosok 1983, 20,16-22,1). ÜS Kraft/Wlosok 1983, 21. 
andere Vergehen vorzugehen oder die tyrannischen Taten der Tetrarchen zu bestrafen, wenn sie sich gegen die Gesamtbevölkerung richten. ${ }^{7}$ Außerdem erfährt der Rezipient

7 Die Tetrarchen machen sich vielfältiger Vergehen gegen die Gesamtbevölkerung schuldig. Siehe beispielsweise das Höchstpreisedikt Diokletians: Tunc ob exigua et vilia multus sanguis effusus, nec venale quicquam metu apparebat, et caritas multo deterius exarsit, donee lex necessitate ipsa post multorum exitium solveretur. Lact. mort. pers. 7,7 (Brandt/Laubmann 1897, 180,22-181,1): „Damals ist wegen unbedeutenden Nichtigkeiten viel Blut vergossen worden, und aus Furcht erschien nichts mehr käuflich, und die Teuerung entbrannte um vieles schlimmer, solange bis das Gesetz aus der Notwendigkeit selbst heraus nach dem Tod vieler abgeschafft wurde“. Vgl. in dieser Arbeit auch Seite 62 und 123; Die Opfergesetze Diokletians: Tunc ira furens sacrificare non eos tantum, qui sacris ministrabant, sed universos, qui erant in palatio, iussit et in eos, si detrectassent, verberibus animadverti datisque ad praepositos litteris etiam milites cogi ad nefanda sacrificia praecepit, ut qui non paruissent, militia solverentur. Lact. mort. pers. 10,4 (Brandt/Laubmann 1897, 184,16-20): „Als er dann vor Zorn raste, befahl er nicht nur, dass diejenigen opfern sollten, die den Opfern als Diener beiwohnten, sondern alle, die sich im Palast befanden, und gab vor, dass gegen diejenigen, wenn sie sich weigerten, mit Schlägen vorgegangen werden sollte und mit Briefen, die an die Vorgesetzten geschickt wurden, dass auch die Soldaten zu den gottlosen Opfern gezwungen werden sollten, mit der Konsequenz, dass diejenigen, die nicht gehorchten, aus dem Militärdienst entlassen würden“. Siehe hierzu Seite 63 und 124 in dieser Arbeit; Der Vergewaltigungstrieb Maximianus': Iam libido in homine pestifero non modo ad corrumpendos mares, quod est odiosum ac detestabile, verum etiam ad violandas primorum filias. nam quacumque iter fecerat, avulsae a complexu parentum virgines statim praesto. Lact. mort. pers. 8,5 (Brandt/ Laubmann 1897, 182,7-11): „Schon war in dem unheilbringenden Menschen die Lust, nicht nur Männer zu verführen, was hässlich und verabscheuenswert ist, sondern auch die Lust, die Töchter der Oberschicht zu vergewaltigen. Denn welchen Weg er auch immer nahm, es wurden die jungen Frauen sofort aus der Umarmung ihrer Eltern entrissen“. Vgl. hierzu Seite 144 in dieser Arbeit; Dignitatem non habentibus poena ignis fuit. Id exitii primo adversus Christianos permiserat datis legibus, ut post tormenta damnati lentis ignibus urerentur. Qui cum delegati fuissent, subdebatur primo pedibus lenis flamma tamdiu, donec callum solorum contractum igni ab ossibus revelleretur. Deinde incensae faces et extinctae admovebantur singulis membris, ita ut locus nullus in corpore relinqueretur intactus. Et inter haec suffundebatur facies aqua frigida et os umore abluebatur, ne arescentibus siccitate faucibus cito spiritus redderetur; quod postremo accedebat, cum per multum diem decocta omni cute vis ignis ad intima viscera penetrasset. Hinc rogo facto cremebantur corpora iam cremata. Lecta ossa et in pulverem comminuta iactabantur in flumina ac mare. Lact. mort. pers. 21,7-11 (Brandt/Laubmann 1897, 197,10 - 22): „Galerius richtet unverhältnismäßige und umfangreiche Strafen gegen alle Bürger: „Für diejenigen, die keine Würde hatten, war das Feuer die Strafe. Diese Art des Todes hatte er zuerst gegen die Christen erlaubt, als er die Gesetze erlassen hatte, dass nach der Folter die Verurteilten vom langanhaltenden Feuer verbrannt werden sollten. Wenn diese festgebunden waren, wurde zuerst eine Flamme unter ihre Füße gelegt solange, bis die harte Haut der Füße vom Feuer zusammengezogen von den Knochen abgerissen wird. Dann wurden angezündete und gelöschte Fackeln an die einzelnen Glieder heran bewegt, sodass keine Stelle am Körper unberührt zurückblieb. Und zwischen diesen Fackeln wurde eiskaltes Wasser über die Körper gespült und der Mund mit Feuchtigkeit abgewaschen, damit nicht durch die Trockenheit den brennenden Schlündern zu schnell der Lebensatem entweiche. Dies geschah zuletzt, wenn über den ganzen Tag hinweg, nachdem die ganze Haut gekocht worden war, die Kraft des Feuers zu den innersten Eingeweiden vorgedrungen war. Dann wurden auf einem aufgestapelten Scheiterhaufen die schon verbrannten Körper verbrannt. Die aufgelesenen und in Staub zertrümmerten Knochen wurden in die Flüsse und das Meer verstreut“. Siehe hierzu Seite 158 in dieser Arbeit; Und auch die nachkriegerischen Streifzüge durch römisches Gebiet seien fatal gewesen: Quod cum timeret, dedit militibus potestatem, ut dispersi quam latissime diriperent omnia vel corrumperent, ut 
zwei weitere Charakteristika Gottes: Er ist ein einziger und ein Richter. Während es nicht spezifisch christlich ist, dass ein Gott straft, richtet oder einen Tempel hat, ist die Aussage, dass er unum sei, zumindest nur noch mit der jüdisch-christlichen Tradition zu verbinden. Kurz darauf spezifiziert Laktanz noch einmal, dass er den christlichen Gott meint. ${ }^{8}$

Gleichzeitig macht Laktanz deutlich, dass die Strafen meritus, verdient, seien. Demnach gibt es ein verbindliches Konzept von Recht und Unrecht, an dem sich menschliche Taten messen lassen. Damit einher geht auch die Zusage, dass jemand, der sich an Gottes Gesetze hält, keine Strafen zu befürchten hat. Für die Christen zur Zeit der Christenverfolgung bedeutet dies aber nicht, dass sie kein Leid zu befürchten hätten. Im Gegenteil, sie müssen Leid über sich ergehen lassen, bis Gott eingreift, und sogar den Tod, beziehungsweise das Martyrium für ihren Glauben in Kauf nehmen.

si quis insequi voluisset, utensilia non haberet. Vastata est igitur ea pars Italiae, qua pestiferum illud agmen incessit, expilata omnia, mulieres corruptae, virgines violatae, extorti parentes et mariti, ut filias, ut coniuges, ut opes suas proderent. Abactae tamquam de barbaris praeda pecorum ac iumentorum. Lact. mort. pers. 27,5-6 (Brandt/Laubmann 1897, 204,16-23): „Weil er dies fürchtete, gab er seinen Soldaten die Möglichkeit, dass sie sich möglichst weit ausstreuend, alles plünderten und vergewaltigten, damit derjenige, falls ihnen irgendwer folgen wollte, keine Vorräte hätte. Also wurde der Teil Italiens völlig verwüstet, durch den dieses unheilbringende Heer zog, alles wurde geplündert, Frauen wurden vergewaltigt, junge Mädchen beschmutzt, Eltern und Ehemänner gefoltert, sodass sie ihre Töchter, ihre Ehefrauen, ihren Besitz hergaben. Weggeführt wurden wie als Beute von Barbaren die Vieh- und Schafherden“. Siehe hierzu Seite 164-166 dieser Arbeit; Auch Daias Hang zu vergewaltigen wirkt sich auf die Gesamtbevölkerung aus Illud vero capitale et supra omnes, qui fuerunt, corrumpendi cupiditas. quid dicam nescio nisi caecam et effrenatam, et tamen his verbis exprimi res pro indignatione sua non potest: vicit officium linguae sceleris magnitudo. Lact. mort. pers. 38,1 (Brandt/Laubmann 1897, 217,4-7): „Jenes war aber das schwerwiegendste, das alle übertraf, die lebten und zwar seine Begierde zu vergewaltigen. Ich weiß nicht, wie ich sie nennen soll, außer blind und zügellos, und dennoch kann die Sache mit diesen Worten nicht ausgedrückt werden wegen ihrer Entrüstung: Die Größe des Verbrechens besiegt die Dienstbarkeit der Zunge“. Siehe auch Seite 171 in dieser Arbeit; eunuchi, leones scrutabantur omnia. ubicumque liberalior facies erat, secedendum patribus ac maritis fuit. detrahebantur nobilibus feminis vestes itemque virginibus et per singulos artus inspiciebantur, ne qua pars corporis regio cubili esset indigna. si qua detrectaverat, in aqua necabantur, tamquam maiestatis crimen esset sub illo adultero pudicitia. aliqui constupratis uxoribus, quas ob castitatem ac fidem carissimas habebant, cum dolorem ferre non possent, se ipsos etiam necaverunt. sub hoc monstro pudicitiae integritas nulla, nisi ubi barbaram libidinem deformitas insignis arcebat. Lact. mort. pers. 38,2-3 (Brandt/ Laubmann 1897, 217,8-218,5): „Eunuchen, ja Zuhälter untersuchten alles. Wo auch immer ein ansehnliches Antlitz war, mussten Vater und Ehemann zur Seite treten. Die Kleider wurden den edlen Frauen weggerissen, sogar den Jungfrauen, und sie wurden Glied für Glied inspiziert, ob irgendein Körperteil dem königlichen Bett unwürdig sei. Wenn eine sich weigerte, wurde sie im Wasser getötet, als ob Keuschheit ein Verbrechen sei unter jenem Ehebrecher. Nach der Vergewaltigung ihrer Frauen, die sie wegen der Schamhaftigkeit und Treue ganz besonders liebten, brachten sich manche selbst um, weil sie den Schmerz nicht ertragen konnten. Unter diesem Monster gab es keine Unversehrtheit der Scham, außer wo besondere Hässlichkeit die barbarische Lust fernhielt“. Siehe auch Seite 172 in dieser Arbeit.

8 Vgl. Lact. mort. pers. 2,1. Laktanz beginnt seine historische Abhandlung mit der Kreuzigung dominus noster Iesus Christus. 
Auch gibt es keinen Anspruch auf göttliches Handeln. Laktanz verhandelt implizit die alttestamentliche Vorstellung des Tun-Ergehen-Zusammenhangs ${ }^{9}$ und zieht die neutestamentliche Erfüllung ${ }^{10}$ eben dieser Verbindlichkeitsvorstellung hinzu. Die theologische Ebene, dass Gott sich dem Menschen gegenüber bei Einhaltung der Gebote daran hält, ihn nicht zu bestrafen, wird hier mit der politischen Vorstellung Laktanz' verknüpft, der zufolge Gott extra terram seine Handlungslogik aufbaut und nicht nach weltlichen Kausalzusammenhängen agiert. Wenn es für Gott richtig ist, für die Exempla seine Strafhandlungen an den Tyrannen zu verschieben, auch wenn es bedeutet, dass Christen leiden müssen, ist es für Gott richtig. Die Gewalt, die Gott an seinen Feinden ausübt, dient dazu, magna et mirabilia exempla zu statuieren, nicht etwa Einzelschicksale zu rächen. Auch in den Gewalttaten zeigt sich also die Größe Gottes und seine Macht.

\title{
5.1 Gottes explizites Handeln
}

Das erste Exempel, das Gott statuiert, betrifft Nero, der die Diener Gottes, Paulus und Petrus, hinrichten ließ:

\begin{abstract}
Nachdem dies Nero berichtet worden war, als er wahrnahm, dass nicht nur in Rom sondern überall täglich eine große Menge vom Kult an den Dämonen abfiel und zur neuen Religion überlief, weil sie die alte verdammte, schritt er, verwünschenswerter und schädlicher Tyrann wie er war, zur Tat, um den himmlischen Tempel auszulöschen und die Gerechtigkeit zu zerstören und als erster von allen die Diener Gottes verfolgend, heftete er Petrus ans Kreuz und tötete Paulus. Dies blieb dennoch nicht ungestraft. Gott schaute nämlich zurück auf die Qualen seines Volkes. Herabgeschleudert also vom Gipfel der Herrschaft und herabgewälzt von der Spitze, wurde der machtlose Tyrann plötzlich unsichtbar, sodass nicht einmal der Ort der Grabstätte eines so wilden Tieres auf der Erde erschien. ${ }^{11}$
\end{abstract}

\footnotetext{
9 Zum Tun-Ergehen-Zusammenhang siehe auch Georg Freuling, Art. Tun-Ergehen-Zusammenhang, in: WiBiLex Onlinelexikon, erstellt 2008, http://www.bibelwissenschaft.de/stichwort/36298/ (zuletzt aufgerufen am 22.09. 2019 12:45 Uhr); Koch (1991). Beispielhaft für die Situation der Christen ist an dieser Stelle sicherlich auch Hiob. Trotz seines vorbildhaften Verhaltens erfährt er für ihn unerklärliche Schicksalsschläge. Dennoch muss er auf Gott vertrauen und dessen Existenz und Liebe nicht in Frage stellen. Vgl. J. Assmann (1990), 203-224; Balzer/Krüger (1997); Selbstverständlich greift der Tun-Ergehen-Zusammenhang nur einen Teilaspekt der alttestamentlichen Theologie heraus.

10 Vgl. Mt. 5,17; hierzu Luz (1978); Deines (2004), besonders 269-279.

11 Qua re ad Neronem delata cum animadverteret non modo Romae, sed ubique cotidie magnam multitudinem deficere a cultu idolorum et ad religionem novam damnata vetusta transire, ut erat execrabilis ac nocens tyrannus, prosilivit ad excidendum caeleste templum delendamque iustitiam et primus omnium persecutus dei servos Petrum cruci adfixit, Paulum interfecit. nec tamen habuit impune. respexit enim deus vexationem populi sui. deiectus itaque fastigio imperii ac devotutus a summo tyrannus impotens nusquam repente comparuit, ut ne sepulturae quidem locus in terra tam malae bestiae appareret. Lact. mort. pers. 2,6-7 (Brandt/Laubmann 1897, 179,9-15).
} 
Nero habe sich gegen Gott gewandt und dies durch die Hinrichtung der Apostel Paulus und Petrus bekräftigt. Zur Strafe sei er von der Erde entrückt worden, sodass kein Grabmal zu finden sei. Nero ist der erste Christenverfolger, den Laktanz beschreibt. Wegen der Christenverfolgungen habe Gott Nero auf dem Gipfel dessen Macht vom Erdboden verschwinden lassen. Gott reagiert auf die grauenvollen Taten Neros und hat Erbarmen mit den Qualen seines Volkes. Gott wird zur Bestrafung auf Satz- wie auf Handlungsebene zum Subjekt. Er schleudere und wälze Nero herab, weil er die Qualen seines Volkes bestrafen wolle. Die Passage ist kurz gehalten, zeigt aber deutlich die gewaltvolle Strafe für den Christenverfolger. Durch die Entrückung Neros wird ihm auch die Grabstätte, sprich die Bestattung verweigert. Die Verweigerung der Bestattung bedeutet für Christen wie Nicht-Christen, dass das Andenken der Nachwelt erschwert wird, wenn nicht sogar verloren geht. ${ }^{12}$ So ist die Entrückung ähnlich einer Damnatio Memoriae, ${ }^{13}$ wenn auch im Falle Neros offensichtlich nicht so effektiv immerhin ist Nero nicht in Vergessenheit geraten, ihm wurde lediglich die Grabstätte als Memorialort verweigert. Statt zu erzählen, was mit Nero geschehen ist, rekurriert Laktanz auf die mythenhafte Erklärung der Zeitgenossen Neros, dass dieser gemäß den Sibyllinischen Orakeln entrückt worden sei, und erklärt, dass dieser Aberglaube unwahr sei und stattdessen Gott Nero ohne Grabmal zu Grunde gerichtet habe. Die Umdeutung dieser mythenhaften Todeserzählung Neros' hilft, die christliche Sichtweise der Vergangenheit zu festigen und kann mit dem Begriff Hans-Joachim Gehrkes als intentionale Geschichte bezeichnet werden. ${ }^{14}$ Auch erklärt die mythifizierte Interpretation der Entrückung, wieso das Geschick Neros nicht als Exempel Gottes erkannt wurde und es wieder zu Verfolgungen kommen konnte.

Der Tod Galerius' ist sicherlich das deutlichste Beispiel für direktes Eingreifen Gottes. Schon zu Beginn der Erzählung erfährt der Leser, dass Gott sich gegen den Tyrannen wende:

Von diesem lenkte Gott, der Rächer seiner religio und seines Volkes, die Augen auf den anderen Maximianus, den Urheber der gottlosen Verfolgung, damit er an ihm auch die Kraft seiner Macht zeigte. $^{15}$

Gott lenke seine Aufmerksamkeit auf Galerius, der als Urheber der Verfolgungen bezeichnet wird. Erstmals wird Gott als vindex (Rächer) bezeichnet. ${ }^{16}$ Er übt Rache an

12 Zur antiken Bestattungsfrage siehe Volp (2002); Erasmo (2008).

13 Zur Damnatio Memoriae siehe beispielsweise und in dieser Arbeit Seite 186-188.

14 Vgl. H.-J. Gehrke (2005), 30 -31; H.-J. Gehrke (2014).

$15 \mathrm{Ab}$ hoc deus religionis ac populi sui vindex oculos ad Maximanum alterum transtulit, nefandae persecutionis auctorem, ut in eo etiam suae vim maiestatis ostenderet Lact. mort. pers. 31,1 (Brandt/ Laubmann 1897, 208,6-8).

16 Die zum Stamm gehörige Verbalform vindicare findet sich acht Mal im gesamten Werk, davon entfällt ein einziges Mal auf den Tätigkeitsbereich Gottes: Lact. mort. pers. 1,7 (Brandt/Laubmann 1897, 173,8). Außerhalb des Proömiums wird die Tätigkeit, sich zu rächen, ausschließlich auf die Tyrannen bezogen (Lact. mort. pers. 28,2 (Brandt/Laubmann 1897, 205,13); 36,1 (Brandt/Laubmann 1897, 214,13); 
denjenigen, die sich an seiner religio ${ }^{17}$ und seinem Volk vergehen, und beschützt die Seinen. Der Begriff vindex bezeichnete oft den Kaiser in seiner besonderen Funktion, den Staat zu schützen, aber auch Rache für Frevel zu üben, war aber auch gebräuchlich für einen Verteidiger vor Gericht. ${ }^{18}$ Wenn Laktanz Gott diese beiden Positionen zuschreibt, unterstreicht er das Handeln Gottes innerhalb der politischen Weltordnung. Auch wenn Gott erst eingreift, sobald die religiöse Sphäre betroffen ist, ist seine Aufgabe doch dieselbe wie die eines Anwalts und Kaisers auf Staatsebene; er rächt und spricht Recht. Nicht alle Herrscher aber werden von Gott eingesetzt oder bestraft, humanpolitische Auseinandersetzungen motivieren ihn nicht zum Handeln. Dies bedeutet auch, dass den Menschen selbst ein gewisser Handlungsspielraum offenbleibt, solange sie die Gesetze Gottes nicht übertreten. Solange die Vergehen sich auf menschliche Belange erstrecken, sind auch menschliche Rechtsinstanzen für Rechtsprechung und Strafe verantwortlich. Für moralisches Fehlverhalten haben die Menschen nach ihrem Tod den Richtspruch zu erwarten, nicht $\mathrm{zu}$ Lebzeiten. ${ }^{19}$ Im Proömium greift Laktanz die Beschreibung Gottes als Rechtsinstanz wieder auf und beschreibt Gott als caelestis iudicis severitas. ${ }^{20}$ Aber auch Daia sehe Gott als iudicans kurz bevor er stirbt:

Dann endlich begann er, nachdem er die Sehkraft verloren hatte, Gott zu sehen, der mit weiß gekleideten Dienern über ihn richtete. Er rief also aus wie diejenigen, die gefoltert werden, es zu tun pflegen, und er sagte, dass nicht er, sondern andere es getan hätten. Dann bekannte er sich, gezwungen von den Qualen, schließlich zu Christus, diesen um Gnade anflehend und bittend, dass er sich seiner erbarmen möge. So hauchte er zwischen den Seufzern, die er, als ob er verbrannt werde, herausbrachte, den schädlichen Geist in der verabscheuungswürdigen Todesart aus. $^{21}$

Maximinus Daia sei erblindet und beginne daraufhin, Gott zu sehen. Gott sitze mit weiß gekleideten Dienern über ihn zu Gericht. Daia versuche sich durch Flehen Gnade

38,5 (Brandt/Laubmann 1897, 218,10-11) ist eine rhetorische Frage, die anzeigt, dass niemand die Taten der Tyrannen rächen könne; 43,4 (Brandt/Laubmann 1897, 222,20); 43,5 (Brandt/Laubmann 1897, 223,3); 44,11 (Brandt/Laubmann 1897, 224,19).

17 Siehe hierzu Seite 84, besonders Anm. 160 in dieser Arbeit.

18 Vgl. Cic. rep. 1,48. Cicero zählt den Schutz des Staates zu den essentiellen Aufgaben eines Herrschers. Gerold Walser legt dar, dass beide Bedeutungsvarianten, Beschützer und Rächer, vorhanden waren. Vgl. Walser (1955).

19 Vgl. Nos igitur melius et verius, qui duas istas vias caeli et inferiorum esse dicimus, quia iustis inmortalitas, iniustis poena aeterna proposita est. Lact. inst. 6,3,10 (Heck/Wlosok 2009, 536,13-15): „Wir wissen es besser und richtiger, die wir sagen, dass es jene zwei Wege zum Himmel und der Unterwelt gibt, weil die Unsterblichkeit den Gerechten, den Ungerechten ewige Strafe auferlegt ist“.

20 Lact. mort. pers. 1,7 (Brandt/Laubmann 1897, 173,8).

21 Tunc demum, amisso visu, deum videre coepit candidatis ministris de se iudicantem. exclamabat ergo sicut ii qui torquentur solent, et non se, sed alios fecisse dicebat. deinde quasi tormentis adactus fatebatur Christum subinde deprecans et implorans, ut suimet misereretur. sic inter gemitus quos tamquam cremaretur edebat, nocentem spiritum detestabili genere mortis efflavit. Lact. mort. pers. 49,5-7 (Brandt/Laubmann 1897, 234,15-21). 
zu verschaffen, bekenne sich zu Christus und sterbe schließlich. Die Informationen über Gott sind auch hier spärlich. ${ }^{22}$ Er richte und sei von Dienern umgeben, die weiß gewandet sind. ${ }^{23}$ Die Urteilsgewalt Gottes steht im Vordergrund. Unabhängig von weltlichen Taten muss sich laut Laktanz jeder Mensch schließlich vor Gott verantworten und dies müsse ihm bereits zu Lebzeiten bewusst sein. Selbst dem Tyrannen Daia, der durch seine Verachtung Gottes und der Christen nicht eingedenk der Konsequenzen seines Handelns ist, wird noch zu Lebzeiten vor Augen geführt, dass er diesem Urteilsspruch unterlegen ist. Laktanz eröffnet durch die Charakterisierung Gottes einen Gegensatz zwischen dem idealen Richter und den tatsächlichen Richtern, beziehungsweise den Tyrannen als Richtern, wie sie im Verlauf des Werkes immer wieder beschrieben werden. Maximianus nutzt die Richter aus, um durch subornatis indiciis die Senatoren umbringen zu lassen:

Und immer, wenn es nötig war, fehlten ihm die reichsten Senatoren nicht, von denen mit untergeschobenen Beweisen behauptet wurde, dass sie nach Herrschaft gestrebt hätten, sodass beständig die Augen des Senats ausgekratzt wurden. Der blutigste Staatsschatz floss über mit Geldmitteln, die auf üble Weise erworben worden waren. ${ }^{24}$

Die Richter, die ein gerechtes Urteil sprechen sollen, sind durch ihre Abhängigkeit von Daia befangen. Sie unterstützen wissentlich die Gewalt gegen Senatoren, indem sie falsche Urteile sprechen. Die Richter sind eine Karikatur ihrer Selbst und arbeiten gegen den Staat, für dessen Erhaltung und reibungsloses Funktionieren sie eintreten sollten. Wie bei den Tyrannen auch, zeigt dieses Verhalten, dass sie das Imperium Romanum trotz ihrer Position im Staatsdienst zugrunde richten statt erhalten wollen. Gleichzeitig übernimmt aber auch Maximinus Daia selbst das Richteramt (iudicare) dafür, was ihn zu einem glücklichen Menschen machen würde:

Dadurch hielt er sich für glücklich. Dadurch glaubte er, dass das Glück seiner Herrschaft bestehen bleibe, wenn er der Wollust und üblen Begierde nichts verweigere. ${ }^{25}$

22 Auffällig ist hier natürlich, dass Laktanz deum videre und anschließend fatebatur Christum nutzt. Um das trinitarische oder dualistische Gottesbild des Laktanz wurde vielfach debattiert Vgl. Heck (2005), 239; Heck (1999), 1044; zum Bild Gottes und der Trinität in den Divinae Institutiones vgl. McGuckin (1983); Siehe auch Wlosok (1970a). In dieser Passage stehen zumindest Gott und Christus scheinbar gleichberechtigt nebeneinander. Die Frage nach dem Gottesbild, besonders in Bezug auf die Trinitätslehre scheint für Laktanz aber noch nicht abschließend geklärt.

23 Vgl. beispielsweise Mk 9,3; 2. Thess 1,6-10; Mt 16,27; Mt 25,31.

24 Et cum opus esset, non deerant locupletissimi senatores qui subornatis indiciis affectasse imperium dicerentur, ita ut effoderentur assidue lumina senatus. cruentissimus fiscus male partis opibus affluebat. Lact. mort. pers. 8,4 (Brandt/Laubmann 1897, 182,4-7). Vgl. Seite 72 und 142 in dieser Arbeit.

25 His rebus beatum se iudicabat, his constare felicitatem imperii sui putabat, si libidini et cupiditati malae nihil denegaret. Lact. mort. pers. 8,6 (Brandt/Laubmann 1897, 182,11-13). Vgl. Seite 74 in dieser Arbeit. 
Er richtet über sich selbst. Wenn der Kaiser derjenige ist, der über sich selbst und seine Handlungen richtet und alle ihm unterstellten Richter sich an dessen Vorgaben halten, bleibt in Gott die einzige Instanz erhalten, die in der Lage ist, Ungerechtigkeiten zu ahnden. Laktanz hat bereits in den Divinae Institutiones dargelegt, wieso das Verhalten Daias, der als epikureischer Philosoph stilisiert wird, nicht dem Verhalten eines Kaisers entspricht. Ein Kaiser, der sich den eigenen Begierden hingibt und darüber die Fürsorge um den Staat vernachlässigt, ist diesem Amt laut Laktanz nicht gewachsen. ${ }^{26}$

Aber auch Diokletian und dessen Richter verurteilten die Christen unrechtmäßig für den Palastbrand:

Er saß selbst bei Gericht vor und röstete die Unschuldigen im Feuer; ebenso quälten auch alle Richter, alle schließlich, die im Palast waren, mit der ihnen gegebenen Macht des Lehrers. Es gab welche, die darüber wetteiferten, wer früher irgendetwas herausfände; nichts wurde jemals herausgefunden, natürlich weil niemand die Angehörigen des Caesaren folterte. ${ }^{27}$

Diokletian vereint die Macht des Kaisers und des Richters in sich. Nachdem er ein Gesetz erlassen hat, kann er so sicherstellen, dass es zu seiner vollen Zufriedenheit umgesetzt wird. Weil er den schieren Massen an Angeklagten mit den tatsächlichen Richtern nicht Herr wird, setzt er außerdem Laien ein, die das Richteramt ausüben sollen. Sein Vorsitz vor Gericht und sein schlechtes Beispiel spornen die eigentlichen Richter dazu an, Unschuldige zu verurteilen und das Richteramt ad absurdum zu führen.

Auch Galerius lasse bei den Steuereintreibungen korrupte Richter einsetzen, die die Steuerlast der Bevölkerung erhöhen mögen:

Demjenigen, der von vielen Wachen umgeben war, blieb keine Möglichkeit, durchzuatmen. $\mathrm{Zu}$ keiner Jahreszeit hatte man auch nur ein bisschen Ruhe. Häufig kam es wegen derselben Personen unter den Richtern selbst oder unter den Soldaten der Richter zu einem Kampf. Kein Platz war ohne Steuereintreiber, keine Weinlese ohne Aufpasser, nichts ließ man den Leidenden zum Leben. ${ }^{28}$

26 Vgl. Lact. inst. 3,17, besonders 17,35-36 und 17,42. Epikur lehre, dass man die Götter nicht fürchten müsse, weil sie sich von menschlichen Angelegenheiten fernhielten. Ohne Furcht vor Repressalien durch Gott gebe es aber keinen Grund, Regeln und Gesetze einzuhalten.

27 Sedebat ipse atque innocentes igne torrebat; item iudices universi, omnes denique, qui erant in palatio, magistri data potestate torquebant. Erant certantes, quis prior aliquid inveniret; nihil usquam reperiebatur, quippe cum familiam Caesaris nemo torqueret. Lact. mort. pers. 14,4-5 (Brandt/Laubmann 1897, 188,1-5). Vgl. Seite 133 in dieser Arbeit.

28 Multis custodiis circumsaepto nulla respirandi facultas, nullo tempore anni vel exigua requies. frequens super isdem hominibus vel ipsis iudicibus vel militibus iudicum pugna. nulla area sine exactore, nulla vindemia sine custode, nihil ad victum laborantibus relictum. Lact. mort. pers. 31,3-4 (Brandt) Laubmann 1897, 208,14-209,6). 
Die Bevölkerung habe keine Möglichkeit, zur Ruhe zu kommen. Die Richter und deren Soldaten seien sich uneinig und die Steuereintreiber seien überall am Werk. Die Richter des Galerius unterstützen seine übermäßige Steuereintreibung statt als Kontrollinstanz im Staat die Bürgerschaft vor unverhältnismäßigen Übergriffen zu schützen. Sie gehen sogar so weit, die Lage der Bürger noch zu verschlimmern. Maximinus Daia hingegen nutze die Richter, um seine Christenverfolgungen weiter voranzutreiben:

Jene sollten aufmerksam sein, damit die Christen weder Versammlungsorte schufen noch sich öffentlich oder privat versammelten, sondern sollten diejenigen, die ertappt worden waren, nach eigener Rechtsprechung zum Opfern zwingen oder den Richtern übergeben. ${ }^{29}$

Die Richter seien für die Umsetzung des Opferedikts zuständig. Laktanz zeigt so, dass unter den Tyrannen das Rechtssystem den Vorstellungen der vorsitzenden Kaiser unterlegen gewesen sei und keineswegs mehr mit den staatlichen Vorgaben übereinstimmte. Wenn Gott also letztlich als Richter vorsitzt, werden die Ungerechtigkeiten derjenigen, die das römische Staatssystem für ihre Zwecke ausgenutzt und pervertiert haben, geahndet. Indem sein Eingreifen aber erst nach dem Tod stattfindet, kann er in seinen Urteilsspruch alle Taten der zu Richtenden einbeziehen. Sein Urteil wird also nicht durch Korruption, ${ }^{30}$ Bitten oder Vergessen beeinflusst. ${ }^{31}$ Keiner der Tyrannen kann sich dem Richtspruch entziehen, auch wenn er durch Bekehrung versuche, Reue zu zeigen. Galerius versuche, durch das Edikt ${ }^{32}$ und die Beendigung der Christenverfolgungen die Krankheit abzuwenden, bleibe hierbei aber erfolglos:

Und dennoch empfing er für diese Tat keine Vergebung für sein Verbrechen von Gott, sondern wurde, wenige Tage nachdem seine Ehefrau und sein Sohn dem Licinius übergeben wurden, als ihm schon die Glieder des ganzen Körpers zerflossen, von grauenhaftem Eiter verzehrt. ${ }^{33}$

29 [Ii] darent operam, ut Christiani neque conventicula fabricarent neque publice aut privatim coirent, sed comprehensos suo iure ad sacrificia cogerent vel iudicibus offerent. Lact. mort. pers. 36,4 (Brandt/ Laubmann 1897, 215,7-9). Vgl. Seite 168 in dieser Arbeit.

30 Zur Korruption im Römischen Reich siehe MacMullen (1988), 84-104.

31 Vergleiche hierzu auch beispielsweise die Wertung Laktanz' in De ira Dei: quid erit homine truculentius, quid imitius, si dempto metu superiore vim legum aut fallere potuerit aut contemnere? timor dei solus est qui custodit hominum inter se societatem, per quem vita ipsa sustinetur munitur gubernatur. Lact. ira 12,4-5 (Kraft/Wlosok 1983, 40,11-14): „Was gibt es nämlich schändlicheres, was herberes als einen Menschen, der, nachdem eine größere Furcht genommen worden ist, die Macht der Gesetze brechen oder verachten kann? Die Angst vor Gott also ist es allein, die die Gemeinschaft der Menschen miteinander schützt, durch die das Leben selbst erhalten, geschützt und gelenkt wird“.

32 Zum Edikt siehe in dieser Arbeit Seite 101-103.

33 Nec tamen ille hoc facto veniam sceleris accepit a deo, sed post dies paucos commendatis Licinio coniuge sua et filio atque in manum traditis, cum iam totius corporis membra diffluerent, horrenda tabe consumptus est. Lact. mort. pers. 35,3 (Brandt/Laubmann 1897, 214,5-8). 
Auch Galerius Bekenntnis zu Christus erlöst ihn nicht, weil seine Vergehen gegen die Christen zu schwerwiegend waren. Als gerechter Richter gibt Gott nicht die Möglichkeit, durch - potentiell sogar vorgeschobene - Bekehrung die Fehler vergessen zu machen. Als Rächer greift er immer dann in weltliches Geschehen ein, wenn er den richtigen Zeitpunkt gekommen sieht. Solange sich die humanpolitischen Auseinandersetzungen nicht auf Christen in ihrem Christsein beziehen, greift Gott nicht ein. Erst wenn die Tyrannen mit der Christenverfolgung beginnen, richtet Gott die Herrscher und bestraft sie mit den für sie grausamsten Strafen. Die Exekutive seiner Judikative überträgt Gott oftmals an seine Stellvertreter.

\subsection{Gottes Stellvertreter und Principes}

Diese Stellvertreter führt Laktanz zu Beginn des Werkes ein und stellt die Prämisse auf, dass die Gewalttaten, die an den Christenverfolgern verübt werden und nicht explizit als Gottes Handeln in Erscheinung treten, trotzdem auf ihn zurückgeführt werden müssen.

Gott hat nämlich Principes eingesetzt, die die gottlosen und blutigen Befehle der Tyrannen ungültig machen und für das menschliche Geschlecht Sorge tragen. ${ }^{34}$

Die Principes seien von Gott eingesetzt und für die Bestrafung der Tyrannen verantwortlich. Hierbei handelt es sich nicht um eine Entscheidung Gottes, die erst zu Zeiten des Laktanz eingesetzt habe, sondern um ein Handlungsmuster, das sich laut Laktanz im Verlauf der Geschichte beobachten lassen kann. Die Christenverfolger vor den Tetrarchen, denen Laktanz die Kapitel 2 bis 6 widmet, wurden bereits durch Stellvertreter hingerichtet. Als erster sei Domitian ${ }^{35}$ durch seine Feinde umgebracht worden und sei anschließend dem Vergessen anheimgefallen:

\footnotetext{
Nachdem aber durch die Eingebung von Dämonen dazu angestachelt wurde, das gerechte Volk zu verfolgen, büßte er bald in die Hände der Feinde übergeben seine Strafen. Aber es war nicht Rache genug, daß er zu Hause umgebracht wurde: Auch die Erinnerung an seinen Namen ist ausgelöscht worden. Denn obwohl er viele bewundernswerte Bauwerke hatte errichten lassen, obwohl er das Kapitol und andere berühmte Denkmäler erbauen ließ, verfolgte der Senat seinen Namen derart, daß nicht einmal eine Spur seines Ehrentitels zurückgelassen wurde. Aufs schwerste brandmarkte der Senat mit Beschlüssen seine Ehre zur ewigwährenden Schande. ${ }^{36}$
}

34 Excitavit enim deus principes, qui tyrannorum nefaria et cruenta imperia resciderunt et humano generi providerunt Lact. mort. pers. 1,3 (Brandt/Laubmann 1897, 172,4-5).

35 Die Christenverfolgung Domitians wird nur von christlichen Autoren behandelt. Siehe hierzu Ulrich (1996); Christ (1962); Jones (1992); Urner (1993).

36 Postquam vero ad persequendum iustum poulum instinctu daemonum incitatus est, tunc traditus in manus inimicorum luit poenas. nec satis ad ultionem fuit quod est interfectus domi: etiam memoria nominis eius erasa est. nam cum multa mirabilia opera fabricasset, cum Capitolium aliaque nobilia monumenta fecisset, senatus ita nomen eius persecutus est, ut neque titulorum eius relinquerentur ulla 
Als Grund für die Christenverfolgung Domitians nennt Laktanz Dämonen ${ }^{37}$, die den Kaiser heimgesucht und ihn zu den Verfolgungen angestiftet hätten. Deren Wirksamkeit schränkt Gott ein, indem er die inimici und den Senat Roms gegen den Verfolger vorgehen lasse. Auch hier unterstreicht Laktanz die zeitliche Verknüpfung von Verfolgung und Niederlage des Tyrannen. Der Tod durch politische Feinde (inimici) im eigenen Land (domi) wiegt besonders schwer, weil er genau wie die Verfügungen des Senats den Kaiser als Tyrannen markiert, der durch Tyrannenmord seines Thrones enthoben wurde und nicht etwa durch Feinde des Staates zu Tode kam wie beispielsweise Valerianus. Die Markierung Domitians als Tyrann durch den Senat zieht auch die Verdammung seines Namens nach sich. Die Vergehen, wegen derer der Senat ihn angeklagt habe, bleiben unbehandelt. Laktanz erweckt den Eindruck, dass auch der Senat auf Gottes Geheiß hin wegen der Christenverfolgungen gegen den Kaiser vorgegangen sei. Die Damnatio Memoriae korrigiert die Rolle, die Domitian im Bild des Reiches durch seine Statuen und Bauwerke eingenommen haben muss. Die Ähnlichkeit zu Diokletian ist groß. ${ }^{38}$ Beide Kaiser haben sich durch ihre umfangreiche Bautätigkeit ausgezeichnet und sind letztlich der Damnatio Memoriae anheimgefallen. Selbstverständlich besteht ein Unterschied darin, dass Diocletian noch gelebt hat und Domitian bereits tot gewesen ist, als sie dieses Schicksal ereilte. Gleichzeitig fällt auch auf, dass Laktanz den Begriff Damnatio Memoriae für Domitian nicht verwendet, sondern nur für Diokletian. Memoria nominis bezeichnet jedoch das gleiche Konzept durch eine Variatio. Gott agiert als politische Instanz und reagiert auf politische Verfehlungen der Tyrannen mit politischen Repressalien, die durch politische Institutionen umgesetzt werden. ${ }^{39}$ Decius sei auf einem Feldzug getötet worden:

Es tauchte nämlich nach sehr vielen Jahren das verfluchte Tier Decius auf, um die Kirche heimzusuchen: Wer nämlich außer einem Schurken würde schon die Gerechtigkeit verfolgen? Und wie wenn er nur deswegen den Gipfel kaiserlicher Macht erklommen hätte, begann er sogleich gegen Gott zu wüten, um sogleich zu stürzen. Denn auf einem Zug gegen die Karpen, die damals Dakien und Mösien besetzt hatten, wurde er sofort von den Barbaren umzingelt und mit einem großen Teil seines Heeres nieder gemacht; nicht einmal die Ehre eines Begräbnisses konnte ihm da zuteil werden, sondern ausgezogen und nackt, wie es sich für einen Feind Gottes gehörte, lag er da, als Futter für wilde Tiere und Vögel. ${ }^{40}$

vestigia, gravissime decretis etiam mortuo notam inureret ad ignominiam sempiternam. Lact. mort. pers. 3,2-3 (Brandt/Laubmann 1897, 177,3-5).

$37 \mathrm{Zu}$ Dämonen bei Laktanz siehe Seite 67-68; Seite 250, besonders Anm. 52 in dieser Arbeit.

$38 \mathrm{Zu}$ den Bauwerken Diokletians siehe Seite 48-50 in dieser Arbeit, zur Damnatio Memoriae Diokletians siehe Seite 186-188 in dieser Arbeit.

39 Die große Ausnahme bilden hier offensichtlich Galerius und Nero, die nicht durch politische Methoden bestraft werden. Vergleiche zu Nero Seite 239-240 und zu Galerius Seite 205-217 in dieser Arbeit.

40 Extitit enim post annos plurimos execrabile animal Decius, qui vexaret ecclesiam: quis enim iustitiam nisi malus persequatur? et quasi huius rei gratia provectus esset ad illud principale fasticium, furere protinus contra deum coepit, ut protinus caderet. nam profectus adversum Carpos, qui tum Daciam Moesiamque occupaverant, statimque circumventus a barbaris et cum magna exercitus parte deletus nec 
Laktanz erwähnt nur, dass der Tod für einen Feind Gottes angemessen sei, nicht jedoch, dass Gott unmittelbar für den Tod verantwortlich sei. Die enge Verknüpfung von Beginn der Verfolgungen und der Niederlage des Kaisers evoziert aber das Bild eines engen kausalen Zusammenhangs in Anknüpfung an die Prämisse des Werkes. Nackt und ausgezogen zu sterben war eine Strafe, die sonst nur von Gladiatorenspielen bekannt war. ${ }^{41}$ Die Verletzlichkeit, die mit der Nacktheit einhergeht, kehrt die Gewalt, die Decius an den Christen, den iusti, verübt habe, ins Gegenteil. Er werde vom Erdboden getilgt und könne keinerlei Begräbnis oder Gedächtnisstätte für sich beanspruchen. Damit einher geht auch der Verlust eines möglichen Weiterlebens nach dem Tod, für einen Kaiser Roms sogar durch Vergöttlichung.

Nach den Christenverfolgern Decius und Domitian beschreibt Laktanz, wie Gott den Tod Valerians veranlasse:

Nicht viel später wurde auch Valerian von nicht unähnlichem Wahnsinn hinfortgerissen und streckte seine gottlosen Hände gegen Gott aus und vergoß, wenn auch nur kurze Zeit, viel gerechtes Blut. Aber jenem ließ Gott mit einer neue und ungehörte Art von Strafe angedeihen, damit er der Nachwelt zum Beweis dafür diene, dass die Gegner Gottes stets die gerechte Strafe für ihre Verbrechen erhalten. Dieser wurde von den Persern gefangengenommen und verlor nicht nur seine Herrschaft, die er unverschämt ausgeübt hatte, sondern verlor auch die Freiheit, die er den übrigen genommen hatte. Er lebte in schändlichstem Sklavendienst. Der Perserkönig Sapor nämlich, der Mann, der ihn gefangengenommen hatte, befahl, dass der Römer sich vor ihm niederknien und den Rücken hinhalten müsse, wenn es ihm beliebte, seinen Wagen oder sein Pferd zu besteigen. Und während er den Fuß auf seinen Rücken setzte, sagte er ihm vorwurfsvoll und unter Gelächter, dass dies die Wirklichkeit sei, und nicht, was die Römer auf Bilder oder Wände malten. [...] Nachdem er aber sein schmachvolles Leben in dieser Schande beendet hatte, wurde ihm die Haut abgezogen und die vom Fleisch abgelöste Hülle mit roter Farbe bestrichen, damit sie im Tempel der Barbarengötter zum Andenken an den ruhmreichen Triumph aufgehängt wird und unseren Gesandten immerfort als Beweis dafür zu dienen, dass die Römer sich nicht zu sehr auf ihre Macht verlassen sollten, weil sie die abgezogene Haut ihres gefangenen Kaisers bei den persischen Göttern besichtigen konnten. ${ }^{42}$

\footnotetext{
sepultura quidem potuit honorari, sed exutus ac nudus, ut hostem dei oportebat, pabulum feris ac volucribus iacuit. Lact. mort. pers. 4,1-3 (Brandt/Laubmann 1897, 178,10-179,2).

41 Vgl. Wiedemann (2001).

42 Non multo post Valerianus quoque non dissimili furore correptus impias manus in deum intentavit [...] hic captus a Persis non modo imerpium, quo fuerat insolenter usus, sed etiam libertatem, quam ceteris ademerat, perdidit vixitque in servitude turpissime. nam rex Persarum Sapor, is qui eum ceperat, si quando libuerat aut vehiculum ascendere aut equum, inclinare sibi Romanum iubat ac terga praebere et imposito pede super dorsum eius illud esse verum dicebat exprobans ei cum risu, non quod in talibus aut parietibus Romani pingeret. [...] postea vero quam pudendam vitam in illo dedecore finivit, derepta est ei cutis et exuta visceribus pellis infecta rubro colore, ut in templo barbarorum deorum ad memoriam clarissimi triumphi poneretur legatisque nostris semper esset ostentui, ne nimium Romani viribus suis fiderent, cum exuvias capti principis apud deos suos cernerent. Lact. mort. pers. 5,1-6 (Brandt/Laubmann 1897, 178,10-179,9).
} 
Gott sei als Subjekt verantwortlich für die Strafe und habe sich einer besonderen Art an Strafe bedient, um ein Exempel für die Nachwelt zu statuieren. Die tatsächliche Ausführung der Strafe erfolgt aber durch den Perserkönig Sapor, der Valerianus foltere. Diese Exempel Gottes hätten nicht ausgereicht, um die Tetrarchen von der Christenverfolgung abzuhalten. Obwohl Gott mit neuen und einzigartigen Strafen agiere, muss die anthropologische Situation der Tyrannen derart sein, dass der Drang zum Bösen überwiege. ${ }^{43}$

Im Vordergrund der Passage steht die Gerechtigkeit Gottes, der semper die verdiente Strafe verhänge. Anhand der Intensität der Strafe lässt sich so auch die Schwere der Verfolgungen ablesen. Wie beim Eingreifen Gottes gegen die Tetrarchen, war schon bei Valerianus die Christenverfolgung Auslöser für Gottes Handeln. Auffallend ist, dass Sapor zum Werkzeug Gottes wird und kein römischer Princeps. Für Laktanz scheint die Frage nach der Überlegenheit des römischen Reiches deutlich hinter Gottes Plan zurückzustehen. Die selbstproklamierte Überlegenheit der Römer wird durch die Beschreibung der Niederlage auf zwei Ebenen weiterentwickelt: Entweder müssen die Römer hinter ihrem tyrannischen Herrscher stehen, der die Christenverfolgungen begangen habe, und dann auch akzeptieren, dass eine fremde Macht, namentlich der Perserkönig, einflussreicher und mächtiger sei und somit das Imperium Romanum dem Perserreich unterlegen sein könnte.

Oder - was Laktanz hier deutlich zu machen versucht - das römische Volk kann sich von ihren Christen verfolgenden Tyrannen lossagen und einsehen, dass der christliche Gott die einzige Instanz ist, die für Gerechtigkeit sorge, auch wenn das bedeute, dass er sich einer den Römern feindlichen Macht bedienen müsse. Da Valerianus isoliert stirbt und das römische Imperium nur nominell eine Niederlage verzeichnen musste, der Darstellung des Laktanz nach jedoch nicht in seinen Grenzen angegriffen wurde, bietet er eine Lesart an, die den christlichen Gott favorisiert und dabei gleichzeitig eine Niederlage der Römer negiert. Die Verknüpfung zwischen dem grausamen Tod Valerianus' und seinen Christenverfolgungen flicht Laktanz in die Beschreibung der Tetrarchen ein, sodass die retroaktive Umcodierung der Todesursache umso deutlicher wird. Die Gewalt, die Valerianus durch Sapor erfährt, ist vornehmlich sozialer Natur, umfasst aber auch schwere körperliche Schläge.

Er verliere die Herrschaft und seine Freiheit, wird also vom Kaiser des Imperium Romanum zum Sklaven degradiert, vom freien, machtvollen Mann zum Unfreien. Diese Unfreiheit wendet Sapor hin zur vollkommenen Un-Menschlichkeit, indem er Valerianus zu einem Fußschemel herabwürdigt. Die Todesszene beschreibt Laktanz nicht, stellt aber heraus, dass die Leiche des Valerianus nach dessen Tod noch ent-

43 Im Kapitel zum Charakter der Tyrannen konnte gezeigt werden, dass der Charakter die Voraussetzung für die Gottesferne der Tyrannen darstellt und ihre Bereitschaft, sich diesen Charaktereigenschaften unterzuordnen der Grund dafür ist, dass sie sich gegen Gott richten. Gewissermaßen unterliegen sie einem Kreislauf: Sie sind charakterlich schwach konstituiert und somit versucht, sich von Gott abzuwenden. Indem sie das tun, erfüllen sie noch weniger ihre menschliche Bestimmung und werden zu Tieren. 
stellt wird. Anders als Nero, ${ }^{44}$ Maximianus ${ }^{45}$ oder Diokletian ${ }^{46}$, soll Valerianus aber gerade nicht der Damnatio Memoriae anheimfallen. Gott lasse Valerianus als Mahnmal im Tempel der heidnischen Götter verbleiben, ${ }^{47}$ er lasse ihn als Exempel für alle Christenverfolger nicht in Vergessenheit geraten. Es lässt sich die Frage stellen, wieso Gott zugelassen hat, dass das Leid des Valerianus einer barbarischen Gottheit zugeschrieben wird und nicht ihm selbst. Es wäre für die Nachwelt schließlich weit deutlicher gewesen, dass die Christenverfolgungen zur Niederlage des Kaisers beigetragen haben, wenn wenigstens nicht ein anderer Gott dafür verantwortlich gemacht worden wäre. Gleichzeitig ist allerdings in der Erklärung des Laktanz völlig unerheblich, wem letztlich der Tod zugeschrieben wird, weil er selbst darlegt, dass der christliche Gott verantwortlich sein muss und der Perserkönig, ein Princeps, wenn auch kein römischer, zu seinen Werkzeugen gehört.

Eine herausragende Position unter den Principes nimmt jedoch Konstantin ein, der durch seine Position als Kaiser für den Tod Maximianus' und Diokletians verantwortlich gezeichnet wird. ${ }^{48}$ Sein überaus mildes Urteil gegen Maximianus, der durch seinen Attentatsversuch auf Konstantin Hochverrat begangen habe, unterstreicht die Herrschertugend clementia Konstantins. Die Strafe, die Gott über Maximianus verhängt, wird also gefiltert durch die Charakterbeschreibung Konstantins. Die bereits im vorangegangenen Kapitel gezeigte Entsprechung von Charakter, Taten und Strafen der Tyrannen verdeutlicht den umfangreichen Blick und die Planung der Strafen, sodass deutlich ist, dass der Tod Maximianus' Entscheidung und Aktion Gottes ist, obgleich die tatsächliche Hand Gottes weder Erwähnung findet noch leicht nachzuvollziehen ist. Die Verwandlung des Tyrannen erinnert stark an die Verwandlungen, die Ovid in seinen Metamorphosen beschreibt. Da dem Rezipienten diese Prätexte bekannt waren, kann er erkennen, dass wie in den Metamorphosen auch hier ein Gott die Verwandlung eines Frevlers herbeiführt. ${ }^{49}$

44 Vergleiche Seite 239-240 in dieser Arbeit.

45 Vergleiche Seite 194-200 in dieser Arbeit.

46 Vergleiche Seite 186-194 in dieser Arbeit.

$47 \mathrm{Zu}$ paganen Gottheiten siehe Seite 250, besonders Anm. 52 in dieser Arbeit.

$48 \mathrm{Vgl}$. Haeret manifestarius homicida et mutus stupet, quasi dura silex aut stet Marpesia cautes. impietatis ac sceleris increpatur. [...] ita ille Romani nominis maximus imperator, qui post longum temporis invervallum cum ingenti gloria viginti annorum vota celebravit, eliso et fracto superbissimo gutture vitam detestabilem turpi et ignominosa morte finivit. Lact. mort. pers. 30,5 - 6 (Brandt/Laubmann 1897, 207,21208,2-5): „Der auf frischer Tat ergriffene Mörder erstarrt und verstummt staunt er, so wie der harte Stein oder die Marpesische Klippe dasteht. Er wird seiner Gottlosigkeit und seines Verbrechens beschuldigt. [...] So beendete jener dem römischen Begriff nach größte Imperator, der nach einer langen Zeitspanne mit unglaublichem Ruhm die Zwanzigjahrfeier beging, nachdem die hochmütigste Kehle zerschmettert und gebrochen worden war, das verabscheuungswürdige Leben durch einen hässlichen und beschämenden Tod“. Siehe in dieser Arbeit Seite 199.

49 Das meiste hierzu wurde bereits im Kapitel zur Gewalt an Tyrannen behandelt. Siehe hierzu Seite 195 in dieser Arbeit. 
Der Tod Diokletians ist ebenfalls vornehmlich durch die Verknüpfung von Vergehen und Art der Strafe miteinander in Verbindung zu bringen. Allerdings findet die Bestrafung Diokletians nicht wirklich durch einen Stellvertreter statt, sondern - wie bereits gezeigt - durch die scheinbar zufällige Verknüpfung der Bestrafung Maximianus durch den Princeps Konstantin und die gemeinsame Abbildung der Tetrarchen. Gott veranlasst Konstantin zur Damnatio Memoriae an Maximianus und straft Diokletian so durch soziale Gewalt, indem er ihm seinen Status als Kaiser aberkennt.

Aber nicht nur die Principes und anderen politischen Akteure handeln im Auftrag Gottes, sondern auch alle anderen Christen seien dazu befähigt. So kann Gott seinen Dienern Kraft geben, Wunder zu wirken:

Während Nero schon herrschte, kam Petrus nach Rom und nachdem gewisse Wunder gewirkt worden waren, die er ja durch die virtus Gottes mit der Kraft, die ihm von ihm selbst gegeben worden war, vollbrachte, bekehrte er viele zur Gerechtigkeit und er errichtete für Gott einen treuen und festen Tempel. ${ }^{50}$

Nimmt Petrus noch eine Sonderrolle unter den Christen ein und ist für die Leser eher als historischer Bezugspunkt zu verstehen, denn als einer von ihnen, bietet Laktanz auch ein Beispiel für die Christen seiner Zeit. Bei den missglückten Opfern Diokletians wirke Gott durch die Menschen, die, bei den Opfern anwesend, die Dämonen vertreiben, die Diokletians Opferschauer angerufen haben:

Als er sich in Teilen des östlichen Reiches aufhielt, als er aus Furcht ein Untersucher künftiger Dinge war, schlachtete er Vieh und suchte in dessen Eingeweiden nach Kommendem. Da machten einige Bedienstete, die um den Herrn wussten, als sie beim Opfern anwesend waren, auf ihre Stirn das unsterbliche Zeichen: Durch diese Tat wurden die Opfer gestört, weil die Dämonen flohen. ${ }^{51}$

Den Christen werde von Gott die Macht über Dämonen ${ }^{52}$ verliehen, die für die paganen Opferschauer selbstverständlich „Götter“ sind. Laktanz wechselt hier die inter-

50 Cumque iam Nero imperaret, Petrus Romam advenit et editis quibusque miraculis, quae virtute ipsius dei data sibi ab eo potestate faciebat, convertit multos ad iustitiam deoque templum fidele ac stabile conlocavit. Lact. mort. pers. 2,5 (Brandt/Laubmann 1897, 174,14-18).

51 Cum ageret in partibus Orientis, ut erat pro timore scrutator rerum futuram, immolabat pecudes et in iecoribus earum ventura quaerebat. tum quidam ministrorum scientes dominum cum adsisterent immolanti, imposuerunt frontibus suis inmortale signum: quo facto fugatis daemonibus sacra turbata sunt. Lact. mort. pers. 10,2 (Brandt/Laubmann 1897, 194,6-11).

52 Der Begriff daemon findet sich auch in Kapitel 3 des Werkes: postquam vero ad persequendum iustum poulum instinctu daemonum incitatus est... Lact. mort. 3,2 (Brandt/Laubmann 1897, 177,3-4): „Nachdem er aber durch die Eingebung der Dämonen dazu angetrieben worden war, das gerechte Volk zu verfolgen“. In den Divinae Institutiones widmet Laktanz den Dämonen größere Aufmerksamkeit. Die Dämonen stammten von gefallenen Engeln ab und würden fälschlicherweise für Götter gehalten. Ihr Ziel sei es aber, die Menschen zu verderben. Vgl. Cum ergo numerus hominum coepisset increscere, providens deus ne fraudibus suis diabolus, cui ab initio dederat terrae potestatem, vel corrumperet ho- 
pretatorische Sichtweise und macht deutlich, dass eine solche Opferschau keine Götter anrufe, wie es im paganen Verständnis unumstößlich gewesen wäre, sondern gerade keine Gottheiten erreichen kann. Diese Logik ergibt sich daraus, dass Gott unus ist. Gott bekräftigt durch diese Machtübertragung seine Anhänger, gegen die Dämonen der Welt eigenmächtig vorzugehen. Er bemächtigt die Christen, die Welt mitzugestalten und das Böse in der Welt zu bezwingen. Wenn Christen auch nicht aus eigenem Antrieb die Tetrarchen zu besiegen in der Lage seien, weil es ihre grundlegende Aufgabe sei, innerhalb des politischen Systems Gehorsam zu leisten, ${ }^{53}$ so seien sie doch zumindest dazu in der Lage, falsche Götter zu erkennen und zu vertreiben. Gott agiere demnach nicht nur selbst und durch hochrangige Principes wie Konstantin und Licinius, sondern durch jeden seiner Anhänger, der sich zu seinem Glauben bekenne und auf Gott vertraue.

mines, vel disperderet, quod in exordio fecerat, misit angelos ad tutelam cultumque generis humani. [Laktanz erzählt Gen 6] sic eos dioabolus ex angelis dei suos fecit satellites ac ministros. qui autem sunt ex his procreati quia neque angeli neque homines fuerunt, sed mediam quandam naturam gerentes, non sunt ad inferos recepti sicut in caelum parentes eorum. ita duo genera daemonum facta sunt, unum caeleste, alterum terrenum. hi sunt immundi spiritus, malorum quae geruntur auctores, quorum idem diabolus est princeps. Lact. inst. 2,14,1-4 (Heck/Wlosok 2005, 185,10-186,5): „Als die Anzahl der Menschen zu wachsen begann, hat Gott in seiner Voraussicht, damit nicht der Teufel, dem er von Beginn an Macht auf der Erde übertragen hatte, seine Menschen durch Betrügereien verderbe oder zerstreue, was er zum Anbeginn getan hatte, Engel geschickt zum Schutz und Sorge um das Menschengeschlecht. [...] So machte der Teufel sie von Engeln Gottes zu seinen Bediensteten und Dienern. Diese aber, die deren Nachkommen sind, weil sie weder Engel noch Menschen sind, sondern eine gewissermaßen in der Mitte gelegene Natur besitzen, wurden nicht in die Unterwelt aufgenommen wie deren Eltern in den Himmel. So wurden zwei Arten von Dämonen geschaffen, eine himmlische und eine irdische. Diese sind Geist der Welt, Urheber der Übel, die begangen werden, und deren Princeps ist ebenso der Teufel“. So gibt es für Laktanz Dämonen irdischen und himmlischen Ursprungs, die für das Unheil der Welt verantwortlich sind. Und zu eben diesen Dämonen muss auch Apollo gezählt werden: de se quidem ille mentitus est, qui cum sit e numero daemonum, angelis se dei adgregavit. Lact. inst. 1,7,9 (Heck/Wlosok 2005, 30,7-8): ,Jener [sc. Apollo] lügt freilich über sich selbst, da er ja in die Schar der Dämonen gehört, sich selbst aber den Engeln Gottes zurechnet“. Die gesamten Dämonen geben sich jedoch als Götter aus: ... illi autem qui desciverunt a dei ministerio, quia sunt veritatis inimici et praevaricatores dei, nomen sibi et cultum deorum vindicare conantur, non quo ullum honorem desideret quis enim perditis honor est? - nec ut deo noceant, cui nocere non potest, sed ut huminibus; Lact. inst. 2,16,9 (Heck/Wlosok 2005, 192, 2-6): ,jene aber, die vom Dienst an Gott abgefallen sind, weil sie Feinde der Wahrheit und Scheinkläger Gottes sind, versuchen für sich selbst den Namen und den Kult der Götter zu beanspruchen, nicht weil sie irgendeine Ehrerbietung wünschen - welche Ehre steht nämlich den Verdorbenen zu? - und nicht um Gott zu schaden, dem nicht geschadet werden kann, sondern um den Menschen zu schaden;“. Das Dämonenverständnis des Laktanz wird ausführlich von Emil Schneweis behandelt.Vgl. Schneweis (1980). Im weiteren Sinne, vor allem aber mit dem Augenmerk auf die Orakel siehe Freund (2006).

53 Siehe hierzu in dieser Arbeit Seite 68, Anm. 100. 


\subsection{Deus universa regens}

Nachdem deutlich geworden ist, welche Handlungen von Gott entweder direkt oder durch seine Stellvertreter ausgeführt wurden, soll nun noch ein Blick auf die Metainformationen geworfen werden. Bei einem Werk über die Todesarten der Christenverfolger, könnte man erwarten, dass Begriffe wie Christ oder Gott entsprechend häufig vorkommen, um den Standpunkt des Autoren deutlich zu machen. Das Gegenteil scheint der Fall: Im gesamten Werk wird der Begriff deus für den christlichen Gott als handelndes Subjekt nur $17 \mathrm{Mal}$ benutzt, ${ }^{54}$ Christus findet insgesamt fünf Mal Erwähnung ${ }^{55}$ und der Begriff Christen immerhin 28 Mal. ${ }^{56}$ Fünf Mal wird „Gott“ davon in den ersten beiden Kapiteln verwendet. ${ }^{57}$ Die vorliegende Analyse der Passagen zeigt aber, dass die von Gott veranlassten Gewalttaten alle Gewalttaten umfassen, die gegen die tyranni oder adversarii Dei gerichtet sind, unabhängig davon, wer sie letztlich ausführt. Auch die vermeintlich freie Entscheidung Maximianus', bei der Wahl seiner Todesart das Erhängen zu bevorzugen, gipfelt in der literarischen Verwandlung des hypermaskulinen Vergewaltigers in eine Frau. Die Entscheidung, welche Art von Gewalt die Tyrannen erfahren, muss immer Gott zugerechnet werden. Wenn aber die Christen in der Lage waren, die falschen Götter zu vertreiben, Gott selbst oder die Principes für den Untergang der Christenverfolger verantwortlich sind, bleibt die Frage offen, wieso die Tyrannen immer noch gegen Gott vorgingen. Laktanz selbst fragt:

Wenn also Gott solche Strafen über die Religionsschänder verhängte, ist es nicht wundersam, dass irgendeiner danach nicht nur so zu handeln wagt, sondern auch gegen die Vormachtstellung des einzigartigen Gottes, der alles lenkt und umfasst, Überlegungen anzustellen? ${ }^{58}$

54 Vgl. Lact. mort. 1,3; 1,4; 1,7; 1,8; 2,7; 5,1; 5,6; 20,5; 31,1; 33,1; 46,5-6 (im Gebet des Licinius vier Mal); 47,3; 49,2; 50,1. In anderen Kasus wird deus immerhin 37 Mal benutzt, davon allein 22 Mal im Genitiv: 1,2; 2,5; 2,6; 3,5 (drei Mal); 4,3; 5,1; 5,7; 6,1; 10,5; 12,2; 15,7; 24,1; 24,5; 33,9; 36,6; 43,1; 44,5; 44,9; 46,3; 50,7; 52,1; 52,4. Im Dativ zwei Mal: 16,6; 25,9, im Ablativ drei Mal: 7,1; 42,3; 52,4 und im Akkussativ zehn Mal: 1,7; 4,2; 5,1; 6,3; 33,9; 34,4; 34,5; 46,3; 49,5; 52,1. Pluralnennungen, die demnach nicht den christlichen Gott bezeichnen können, gibt es drei im Genitiv: 5,6;11,1;11,6 und fünf im Akkussativ: 5,6;11,7;15,5; 17,5; 36,4 .

55 Vgl. Lact. mort. 2,1; 2,9; 16,9; 44,5; 49,6, davon einmal im Nominativ (2,1), zwei Mal im Akkussativ $(44,5$ und 49,6) und zwei Mal im Genitiv (2,9 und 16,9). Besonders spannend sind die Genitivverbindungen, von denen die erste das imperium Christi sanctum ac sempiternum beschreibt und die zweite auf den militem Christi verweist. Eine genauere Analyse der Begriffe, ihrer Häufigkeit und ihres Verwendungskontexts stellt ein weiteres Forschungsdesiderat dar.

56 Vgl. Lact. mort. 10,6; 11,1; 11,6; 14,2 (zwei Mal); 21,7; 22,1; 24,9; 34,1 (zwei Mal) und 34,4 (im Edikt des Galerius); 36,3 (zwei Mal); 36,4; 46,2; 48,2-10 (zwölf Mal in der Verlautbarung Konstantins und Licinius').

57 Vgl. Lact. mort. 1,$3 ; 1,4 ; 1,7 ; 1,8 ; 2,7$.

58 Cum igitur tales poenas de sacrilegis deus exegerit, nonne mirabile est ausum esse quemquam postea non modo facere, sed etiam cogitare adversus maiestatem singularis dei regentis et continentis universa? Lact. mort. pers. 5,7 (Brandt/Laubmann 1897, 179,9-13). 
Diese rhetorische Frage zielt nicht auf die Kompetenz Gottes ab, sondern darauf, zu zeigen, dass die Tetrarchen besonders schlimme Tyrannen seien. Dennoch erfährt der Rezipient, dass Gott universa regens ac continens sei. Und eben dieser einzige, alles umfassende und lenkende Gott war für jede einzelne Qual der Christenverfolger verantwortlich. Jede Gewalttat, die Gott an den Tyrannen verübt, ist gerechtfertigt und auf Charakter und Taten der Verfolger abgestimmt, auch wenn die Exempla, die Gott statuiert, eines christlichen Autors wie Laktanz bedürfen, um als solche erkennbar zu werden. Sowohl christliche als auch pagane Rezipienten können nun nicht mehr umhin, das Handeln Gottes in der Welt zu begreifen und die Konsequenzen für Christenverfolgungen in der Geschichte manifestiert zu erkennen. 